\title{
Accurate quantification of dystrophin mRNA and exon skipping levels in Duchenne Muscular Dystrophy
}

\author{
Pietro Spitali ${ }^{1,2}$, Hans Heemskerk ${ }^{1}$, Rolf HAM Vossen ${ }^{1}$, Alessandra Ferlini ${ }^{2}$, Johan T den Dunnen ${ }^{1}$, Peter AC 't Hoen ${ }^{1}$ \\ and Annemieke Aartsma-Rus ${ }^{1}$
}

Antisense oligonucleotide (AON)-mediated exon skipping aimed at restoring the reading frame is a promising therapeutic approach for Duchenne muscular dystrophy that is currently tested in clinical trials. Numerous AONs have been tested in (patient-derived) cultured muscle cells and the $m d x$ mouse model. The main outcome to measure AON efficiency is usually the exon-skipping percentage, though different groups use different methods to assess these percentages. Here, we compare a series of techniques to quantify exon skipping levels in AON-treated $m d x$ mouse muscle. We compared densitometry of RT-PCR products on ethidium bromide-stained agarose gels, primary and nested RT-PCR followed by bioanalyzer analysis and melting curve analysis. The digital array system (Fluidigm) allows absolute quantification of skipped vs non-skipped transcripts and was used as a reference. Digital array results show that $1 \mathrm{ng}$ of $m d x$ gastrocnemius muscle-derived mRNA contains $\sim 1100$ dystrophin transcripts and that 665 transcripts are sufficient to determine exon-skipping levels. Quantification using bioanalyzer or densitometric analysis of primary PCR products resulted in values close to those obtained with digital array. The use of the same technique allows comparison between different groups working on exon skipping in the $m d x$ mouse model.

Laboratory Investigation (2010) 90, 1396-1402; doi:10.1038/labinvest.2010.98; published online 10 May 2010

KEYWORDS: antisense oligonucleotides; digital array; duchenne muscular dystrophy; exon skipping; transcript quantification

Antisense oligonucleotide $(\mathrm{AON})$ treatment is one of the most promising therapeutic approaches for Duchenne muscular dystrophy (DMD) and involves the use of chemically modified RNA molecules complementary to pre-mRNA to hide exons from the splicing machinery and restore the disrupted open reading frame, allowing the generation of partly functional instead of non functional dystrophin. ${ }^{1}$ Single or double exon skipping can be used to 'reframe' out-of-frame deletions, duplications and small mutations. ${ }^{2}$ Numerous groups are working on this therapeutic approach using antisense oligonucleotides (AONs) carrying different chemical modifications. The $2^{\prime}$-O-methyl phosphorothioate (2OMePS) and morpholino phosphorodiaminate oligomer chemistries are used most and proof-of-concept has been obtained for both chemistries in clinical trials where patients were locally injected in tibialis anterior and EDB muscles, respectively. $^{3,4}$

Exon skipping is a personalized-medicine type approach: patients with different mutations need skipping of different exons to 'reframe' their out-of-frame dystrophin transcript. Although skipping of certain exons would be applicable to larger cohorts of patients-eg, skipping of exon 51 can be used to 'reframe' the transcript in $13 \%$ of Duchenne Muscular Dystrophy (DMD) patients ${ }^{2}$ — the need to skip different exons to treat larger groups of patients, requires a reliable and comparable outcome to assess the efficacy of different RNA antisense molecules. The most commonly used parameter when comparing AONs is the exon-skipping percentage. This is defined as the percentage of transcripts in which the targeted exon is skipped relative to the total number of dystrophin transcripts (skipped and non skipped). As anticipated, there appears to be a correlation between exon-skipping percentages and dystrophin restoration in that AONs that induce high levels of exon skipping also lead to higher dystrophin levels than AONs that are less efficient. ${ }^{5}$ Thus, when optimizing AONs, assessment of exon skipping percentages is a relatively straightforward way to compare the efficiencies of different AONs. However, different ways to

\footnotetext{
'Department of Human Genetics, Leiden University Medical Center, Leiden, The Netherlands and ${ }^{2}$ Department of Experimental and Diagnostic Medicine, Section of Medical Genetics, University of Ferrara, Ferrara, Italy

Correspondence: Dr P Spitali, PhD, Department of Experimental and Diagnostic Medicine, Section of Medical Genetics, Via Fossato di Mortara, 74, 44100 Ferrara, University of Ferrara, Italy. E-mail: pietro.spitali@unife.it

Received 23 December 2009; revised 25 March 2010; accepted 31 March 2010
} 
quantify exon-skipping levels are used by different groups, making it difficult to compare results between labs. ${ }^{5-11}$

As dystrophin expression is rather low, cDNA synthesis followed by two rounds of PCR amplification (primary and nested PCR) is the method used most to detect and quantify the exon-skipping percentage, but protocols greatly differ and the total number of amplification cycles can vary from $\sim 50$ to $70 .^{5,7-11}$ Only one group has recently used a single round amplification ${ }^{12}$ to assess exon 23 skipping in the $m d x$ mouse, which is a dystrophin-negative mouse model with a point mutation in the in-frame exon 23 and which has been instrumental to optimize the exon-skipping approach in vivo. Although RT-PCR-based methods are inexpensive and allow high throughput, there is a possibility that the shorter skipped fragment is amplified more efficiently than the larger unskipped fragment, thus leading to an overestimation of exon-skipping percentages. Relative quantification of RT-PCR products can be performed using bioanalyzer technology to assess exon-skipping percentages. ${ }^{5,6}$ Alternatively, densitometric analysis to assess the intensity of fragments in the agarose gel has been used. ${ }^{10}$ This could, however, lead to a bias since the longer unskipped fragments bind more intercalating ethidium bromide than the shorter skipped fragments, leading to a brighter signal and underestimation of exon-skipping percentages.

We introduce here as new methods to measure exon 23 skipping in the $m d x$ mouse model a melting curve analysis (MCA) and a digital array by Fluidigm. MCA allows discrimination and relative quantification of different PCR products. ${ }^{13,14}$ Amplicons are heated gradually to melt out the fragments, resulting in the release of the intercalating dye. This creates a melting curve that is a function of the amplicon sequence. Generally a single base pair difference between amplicons leads to an altered melting curve. The area under the curve can be used for relative quantification and MCA can be used to analyse multiple samples at the same time. Quantification is fluorescence based and this method is less expensive than TaqMan technology fluorescence-based methods because an intercalating dye (eg, LCgreen) is used. However, this comes at the cost of reduced specificity when compared with TaqMan probes.

The digital array approach ${ }^{15}$ is based on TaqMan probes and allows an absolute quantification of two RNA species underlining two main outcomes: (i) the determination of the amount of dystrophin transcripts per ng of RNA (total and/ or mRNA) allowing for calculation of the number of templates present when performing the RT-PCR and (ii) the identification of two RNA species (skipped and unskipped) to determine the absolute exon-skipping percentages. In this way, one can compare results between labs and/or between experiments performed at different time points. Although an absolute quantification method, such as the digital array, represents the golden standard, it is more expensive than the other methods.
We here compared six different methods to assess exonskipping percentages, including a digital array, which was used as a golden standard because it allows absolute quantification, to identify the most accurate, affordable and straightforward method.

\section{MATERIALS AND METHODS RNA Isolation and CDNA Synthesis}

Samples used in this study have been described previously. ${ }^{5}$ Briefly, $7 \mathrm{mdx}$ mice were used that were injected in the gastrocnemius muscle on 2 consecutive days with $2.9 \mathrm{nmol}$ of either morpholino or 2-OMePS AON (approximately $20 \mu \mathrm{g}$ ) at 4-5 weeks of age. They were killed by cervical dislocation 10 days after the last injection. Treated muscles were isolated and minced in RNA-Bee (Campro Scientic, Veenendaal, The Netherlands) using MagNa Lyser green beads (Roche Diagnostics, Mannheim, Germany) and the MagNa Lyser (Roche Diagnostics) according to the manufacturer's instructions. cDNA was generated using $400 \mathrm{ng}$ total RNA with random hexamer primers using Transcriptor reverse transcriptase (RT) (Roche Diagnostics) according to the manufacturer's instructions.

\section{Digital Array}

cDNA from 12 samples was loaded onto the digital array (Fluidigm, Bioké, Leiden, The Netherlands). Each sample was partitioned in 765 reaction chambers of $6 \mathrm{nl}$ each, in which the PCR mix was already present. Primers in exon 22 and exon 24 (Eurogentec, Liege, Belgium) amplified both skipped and unskipped molecules, and two different TaqMan MGB probes (Applied Biosystems, Applera Italia, Monza, Italy) recognized either exon 23 (FAM-labeled) or the exon22exon24 junction (VIC-labeled) (primer and probe sequences available in Supplementary Information). Samples were diluted so that 0 or 1 molecule of cDNA template was present in a single amplification chamber. Thus, the percentage of exon skipping can be calculated by counting the number of FAM and VIC-positive spots. For each sample loaded we used cDNA derived from $\sim 7.5 \mathrm{ng}$ total RNA template. The final concentration of primers and probes were $1.125 \mu \mathrm{M}$ and $0.25 \mu \mathrm{M}$, respectively.

\section{Primary and Nested PCRs}

PCRs were performed by 30 cycles of $94^{\circ} \mathrm{C}$ for $30 \mathrm{~s}, 60^{\circ} \mathrm{C}$ for $30 \mathrm{~s}$ and $72^{\circ} \mathrm{C}$ for $30 \mathrm{~s}$ in a $50 \mu \mathrm{l}$ reaction using $1.5 \mu \mathrm{l} \mathrm{cDNA}$ as template. PCR products were visualized on $2 \%$ agarose gels and quantified either with densitometric analysis (QuantityOne-Biorad) or the Agilent 2100 Bioanalyzer (Agilent, Santa Clara, CA, USA).

For nested PCRs, a primary PCR was performed for 19 cycles of $94^{\circ} \mathrm{C}$ for $40 \mathrm{~s}, 60^{\circ} \mathrm{C}$ for $40 \mathrm{~s}$ and $72^{\circ} \mathrm{C}$ for $120 \mathrm{~s}$ in a $25 \mu \mathrm{l}$ reaction using $3 \mu \mathrm{l}$ of cDNA as template. The amplified fragments were then diluted 10 times and $1 \mu \mathrm{l}$ was re-amplified in a nested PCR using internal primers 
(see Supplementary information) for 32 cycles of $94^{\circ} \mathrm{C}$ for $40 \mathrm{~s}, 60^{\circ} \mathrm{C}$ for $40 \mathrm{~s}$ and $72^{\circ} \mathrm{C}$ for $100 \mathrm{~s}$.

PCR products were visualized and analyzed as described for the primary PCRs.

\section{Melting Curve Analysis}

Skipped and unskipped fragments were isolated from agarose gel, quantified using the Nanodrop ND1000 spectrophotometer (Isogen, de Meern, the Netherlands) and used as template for subsequent amplification and melting curve analysis. Skipped and unskipped molecules were mixed in known ratios, ranging from 0 to $100 \%$ skipping based on the number of molecules, and used as template in a 30 cycle amplification (see RNA isolation and cDNA synthesis), using LC-green (Bioké, Leiden, the Netherlands) as intercalating dye. Melting curves were generated with the Lightscanner (Roche Diagnostics, Almere, the Netherlands).

\section{Amplification of Skipped and Unskipped Molecules in Known Molecular Ratios}

Skipped and unskipped templates were isolated and mixed in known molecular ratios using 20-25 ng of template. PCRs were performed with 20,25 or 30 cycles of $94^{\circ} \mathrm{C}$ for $30 \mathrm{~s}$, $60^{\circ} \mathrm{C}$ for $30 \mathrm{~s}$ and $72^{\circ} \mathrm{C}$ for $30 \mathrm{~s}$ in $25 \mu \mathrm{l}$ reaction with $5 \mu \mathrm{l}$ of template.

\section{Statistical Analysis}

Stata software was used for statistical analysis. The Friedman test was used to detect differences between all groups and the Wilcoxon's signed rank sum test was used to compare the different groups from the digital array group.

\section{RESULTS}

Here, we compared six different methodologies (digital array, densitometry and bioanalyzer of primary and nested RT-PCR products and MCA) to quantify exon-skipping percentages. Most dystrophin exon skipping experiments are performed in the $m d x$ mouse to optimize systemic dosing regimes using AONs targeting mouse exon 23. Thus, we quantified murine exon 23 skipping on RNA obtained from AON-treated $m d x$ muscle tissues. ${ }^{5}$

In all, $400 \mathrm{ng}$ of total RNA isolated from AON-treated muscles with varying skipping levels was used to synthesize cDNA, which was subsequently used as input for the different analyses.

\section{Digital Array}

The digital array allows absolute quantification of exon skipped and wild type transcripts.

The output of this experimental approach allows the determination of the number of transcripts per ng of RNA as well as the exon-skipping percentage.
First, amplification was performed on the 480 Roche Light Cycler using either skipped or unskipped templates. This confirmed the specificity of the designed real-time probes (data not shown).

Fluorescent signals of skipped and unskipped transcripts are visible using the Fludigm digital array machine in the FAM and VIC channels, respectively (Figure 1). Normal (unskipped) and skipped identifying probes were used, and some low level VIC to FAM bleeding was observed (Supplementary Figure 1a). Therefore, a threshold to correct for this was applied. Automated counting of the absolute number of FAM- and VIC-positive amplification chambers allowed us to calculate the exon-skipping percentages listed in Table 1 and graphed in Figure 2. Exon skipping levels varied between $4 \%$ and $32 \%$ for the different samples.

The absolute quantification allowed us, for the first time, to calculate the number of dystrophin transcripts present in muscle. The average number of transcripts (skipped and unskipped) per ng of total RNA input for the seven samples was $22.15( \pm 3.30)$. Considering mRNA is estimated to be $\sim 2 \%$ of total RNA (http://www.ambion.com/techlib/basics/ rnaisol/index.html), the number of dystrophin transcripts per ng of mRNA is $1108(50 \times 22.15)$. Using this number we determined the number of transcripts used as template by different groups that studied exon 23 skipping in the $m d x$ mouse model (Table 2). This number varied from $1100^{16}$ to $17700 .^{8}$

\section{RT-PCR and Densitometry}

A total of 665 template molecules were used for one round of amplification and samples were analyzed with ethidiumbromide stained agarose gels (Figure 3a). Exon skipping percentages were assessed with a densitometer (Table 1, Figure 2). They were very close to those obtained using digital array for all analyzed samples and for four out of seven samples the skipping percentages were falling in a $25 \%$ interval relative to the digital array output. We then performed a two-round amplification (nested PCR) and assessed exon skipping percentages (Figure $3 \mathrm{~b}$ ). Now, exon skipping levels were 1.4- to 7.6-fold higher than the digital array reference values and the densitometric analysis of a single round of amplification (Figure 2 and Table 1).

\section{RT-PCR and Bioanalyzer Analysis}

The same fragments obtained after the primary and nested PCRs (Figure 3a and b) were used to quantify exon skipping levels using the Agilent Bioanalyzer (Figure 2 and Table 1). As with densitometry, exon skipping levels after a single round of amplification were very similar to those obtained with the digital array and for five out of seven samples the skipping percentages were falling in a $25 \%$ interval relative to the digital array output. For nested PCR the exon skipping levels were 1.6- to 5.8-fold higher compared with digital array outcomes and to primary PCR values. 


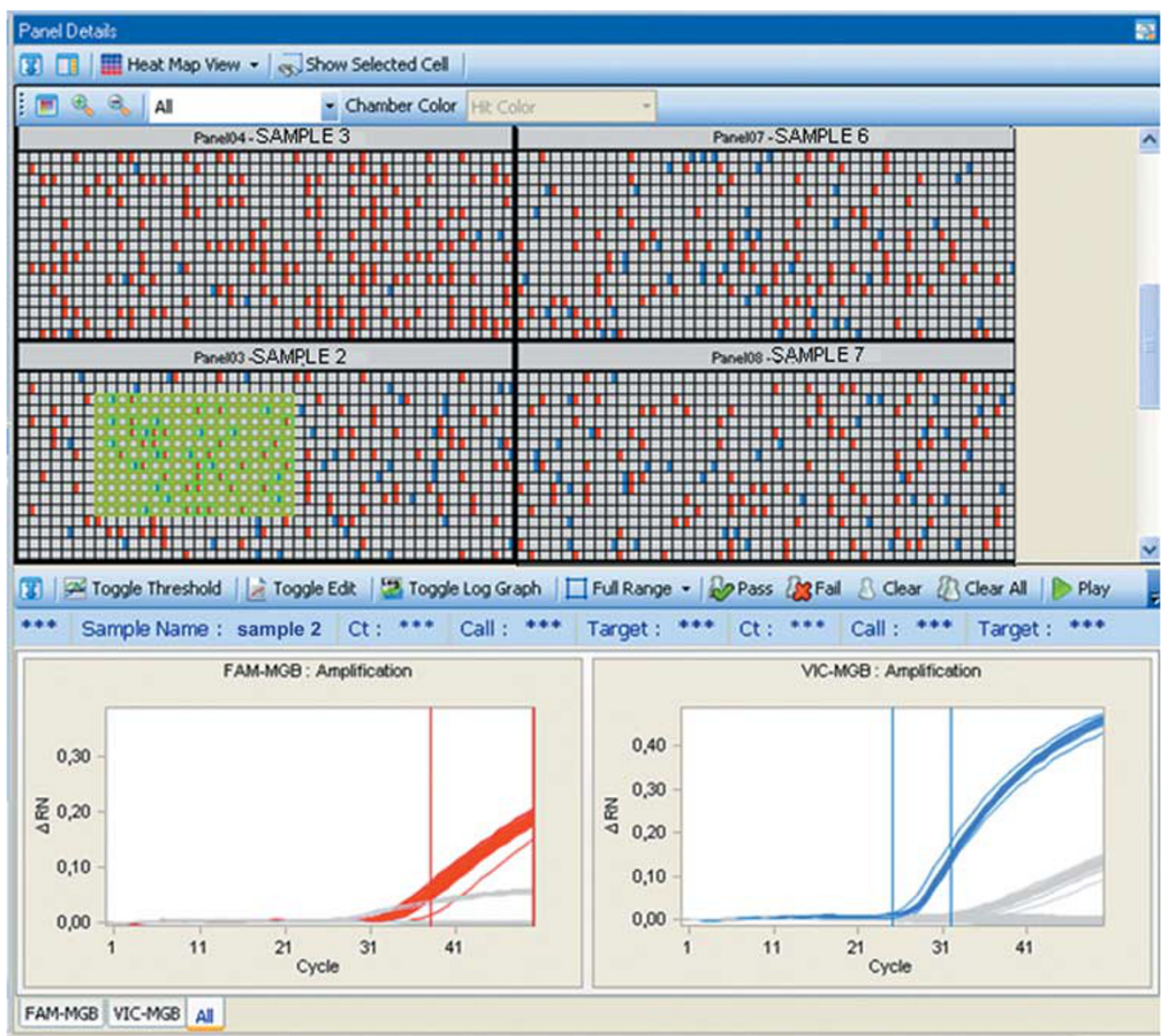

Figure 1 Digital array output for samples 2, 3,6 and 7. Red chambers are FAM positive, blue ones are VIC positive and gray ones are negative for both. When a selected area is analyzed (eg, green area in the bottom left panel) the fluorescent signals are shown in the FAM and VIC channels. Amplification curves are easily recognizable over the background signals for both FAM and VIC signals.

Table 1 Exon skipping percentages using the different quantification methods

\begin{tabular}{|c|c|c|c|c|c|}
\hline Samples & Digital array & Densitometer primary & Lab-chip primary & Densitometer nested & Lab-chip nested \\
\hline 1 & $30.8 \pm 3.8$ & $24.8 \pm 0.5$ & $29.2 \pm 0.6$ & $42.0 \pm 1.9$ & $48.3 \pm 1.5$ \\
\hline 2 & $32.0 \pm 4.1$ & $31.3 \pm 0.7$ & $38.7 \pm 0.8$ & $45.3 \pm 4.0$ & $57.3 \pm 3.9$ \\
\hline 3 & $4.3 \pm 1.0$ & $8.4 \pm 0.2$ & $7.1 \pm 0.1$ & $32.8 \pm 1.5$ & $24.9 \pm 1.3$ \\
\hline 4 & $4.6 \pm 1.1$ & $3.3 \pm 0.1$ & $4.1 \pm 0.1$ & $32.5 \pm 2.5$ & $19.9 \pm 7.4$ \\
\hline 5 & $9.9 \pm 1.5$ & $6.1 \pm 0.1$ & $6.3 \pm 0.1$ & $31.9 \pm 1.5$ & $20.9 \pm 1.8$ \\
\hline 6 & $28.6 \pm 3.5$ & $28.3 \pm 0.6$ & $33.8 \pm 0.7$ & $44.5 \pm 0.5$ & $49.9 \pm 0.8$ \\
\hline 7 & $27.9 \pm 3.6$ & $30.3 \pm 0.6$ & $34.7 \pm 0.7$ & $40.1 \pm 1.1$ & $49.9 \pm 0.5$ \\
\hline
\end{tabular}

Percentages falling within a $25 \%$ interval (relative to the digital array values) are highlighted in gray. Experiments were performed in duplicate.

\section{Melting Curve Analysis}

Skipped and unskipped templates mixed at known molarities were amplified using a saturating intercalating dye (LC-green) and PCR products were melted out. This resulted in specific amplification curves for both unskipped and skipped templates (Supplementary Figure 2). However, when templates were mixed we were unable to discriminate between 10 and $20 \%$ skipping, between 30 and $40 \%$ skipping or between curves ranging from 60 to $100 \%$ skipping (Supplementary Figure 2). We discarded this method because of the low sensitivity.

\section{Amplification of Skipped and Unskipped Molecules in Known Molecular Ratios}

Finally, to confirm there was no preferential amplification of shorter (skipped) fragments in a 30 cycle PCR, skipped and 


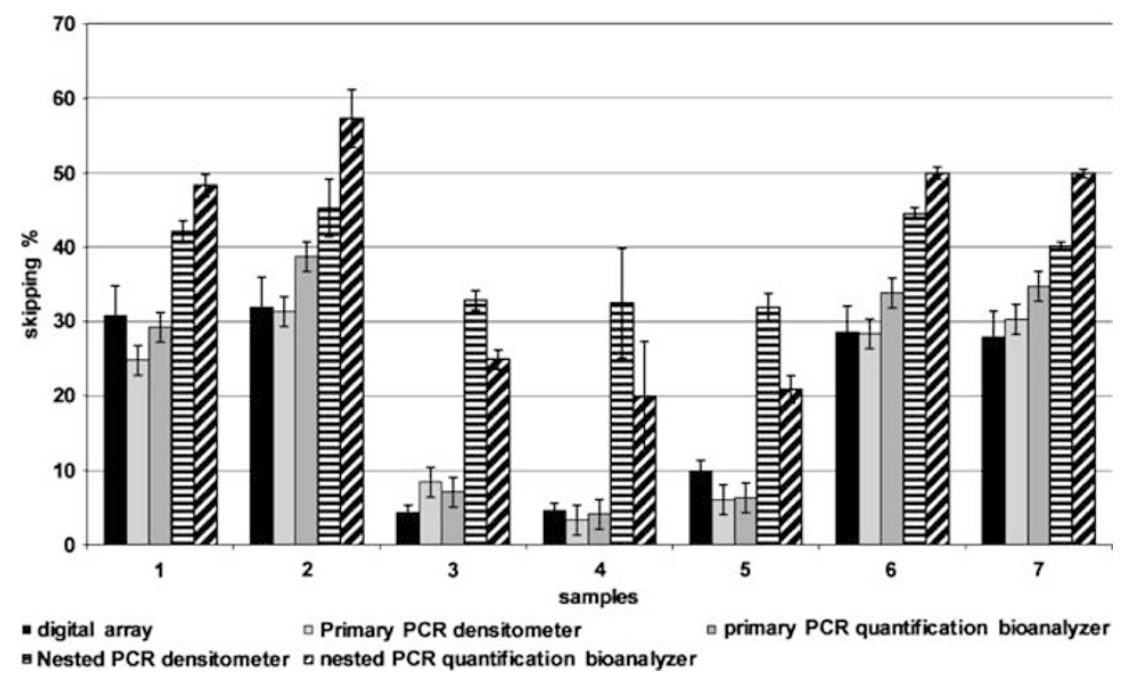

Figure 2 Graphic representation of the mouse exon 23 skipping percentages assessed using the different techniques. Densitometry and bioanalyzer relative quantification output was closest to the digital array results. Experiments were performed in duplicate.

Table 2 Comparison of used amplification protocols to detect exon 23 skipping in the mdx mouse model

\begin{tabular}{|c|c|c|c|c|c|c|c|c|c|}
\hline No. of cycles & 30 & 40 & $(25+25)$ & $(25+25)$ & $(19+32)^{\mathrm{a}}$ & $(30+25)$ & $(30+30)^{a}$ & $(35+30)$ & $(40+30)^{b}$ \\
\hline RNA template ng in $\mathrm{RT}$ reaction & 400 & 100 & 200 & 800 & 400 & 200 & 500 & 100 & 200 \\
\hline RNA template ng in PCR & 30 & 100 & 200 & 800 & 60 & 200 & 50 & 100 & 200 \\
\hline
\end{tabular}

Nanograms of total RNA template used for RT reaction and primary PCR (when different) are shown. The number of transcripts used as templates for the primary PCR as template is also shown. The last row shows the additional amount of amplification compared with the 30 cycles protocol used in this report.

a Before nested amplification DNA template was diluted 10 times.

$\mathrm{b}_{4} \mu \mathrm{l}$ of DNA template were used in nested PCR.
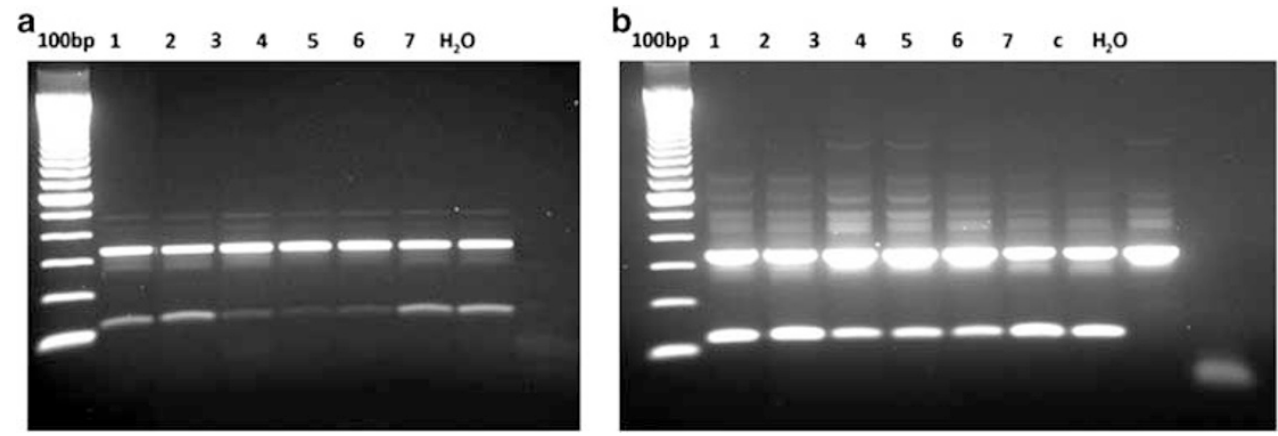

Figure 3 RT-PCR analysis of dystrophin mRNA fragments of $m d x$ mouse muscles treated with different 2OMePS and morpholino AONs targeting exon 23. (a) Fragments obtained after 1 round of amplification. (b) Fragments obtained after 2 rounds of amplification.

unskipped templates were mixed in known ratios based on the molecule numbers and amplified for 20 or 25 or 30 cycles. Bioanalyzer measured skipping values (Supplementary Figure 3) were comparable with the expected ones in the range of $0-50 \%$ skip. The measured skip percentages were a slight underestimation compared with the excepted percentages (Figure 4), suggesting there is no preferential amplification of the shorter products.

\section{Statistical Analysis}

Exon skipping values obtained with each technique were first analyzed by grouping the skipping values of all the mice 


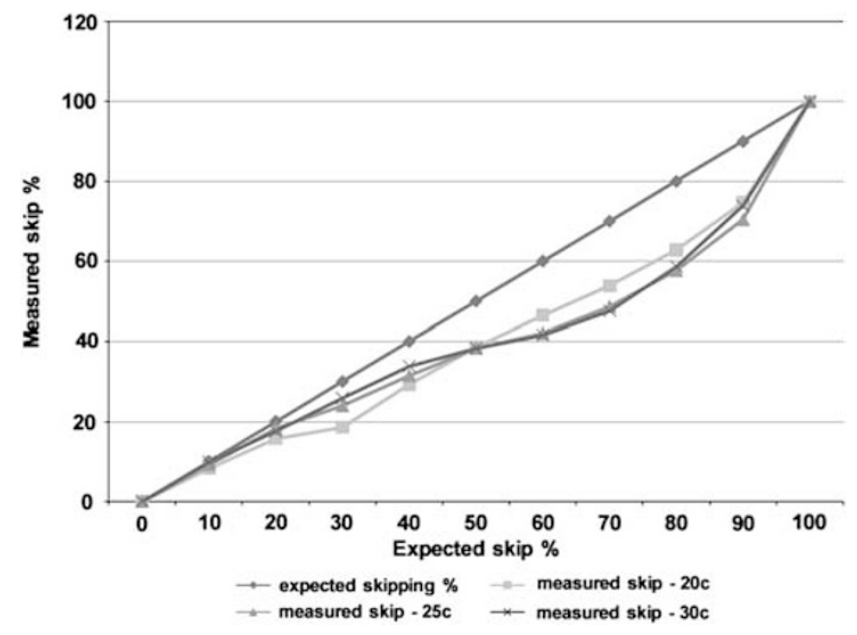

Figure 4 Graphic representation of the bioanalyzer results for 20/25/30 cycles of amplification of skipped and unskipped fragments mixed in different ratios ranging from 0 to $100 \%$ skip. Measured skip percentages were never above the expected ones.

obtained. We found a significant difference between all the groups ( $P$-value 0.0002 , Friedman's test). When single groups were compared with the digital array group we found that the output of the two nested-PCR quantification groups was significantly different from the digital array output $(P$-value $<0.02$ Wilcoxon's test), whereas the skipping values obtained using the primary PCR were not different to the digital array.

\section{DISCUSSION}

AON-mediated exon skipping is currently tested in clinical trials $^{3,4}$ and has proven to be one of the most promising therapeutic strategies to treat DMD. ${ }^{17}$ When optimizing AON chemistries, cell penetrating peptide-conjugates ${ }^{7}$ or AON delivery compounds, the main outcome to evaluate the treatment efficiency is generally the exon-skipping percentage. This can and has been measured using different techniques during the past decade. ${ }^{5,9,11,18,19}$ The most commonly used method is a two-round amplification (nested PCR) of cDNA obtained from an RT reaction using different amounts of total RNA (100 to $800 \mathrm{ng}^{8,11}$ ) as template. To assess the optimal method to determine exon-skipping percentages, we reviewed the most used methods and compared them using AON-treated mouse muscle-derived RNA as input material. Digital array allowed us to quantify the absolute exonskipping percentages (2nd column in Table 1) and was used as the golden standard for the other five methods.

The digital array output also allowed us to determine that $\sim 1100$ dystrophin transcripts are present in $1 \mathrm{ng}$ of mRNA and that 665 transcripts are sufficient to correctly determine the exon-skipping percentage using only 30 cycles of amplification. These data match with the data published by Mortazavi et $a l,{ }^{20}$ in which an RNA-Seq experiment was performed in (among others) skeletal muscle tissue of a wild-type mouse. This resulted in dystrophin expression of
1.53 reads per kilobase per million, which corresponds to 1935 transcripts per ng of mRNA (assuming $1.4 \mathrm{~kb}$ is the average size of an mRNA molecule ${ }^{21}$ ). The two results, although slightly different, are in the same order of magnitude and the difference could be caused by for example, reduced transcript levels due to nonsense-mediated mRNA-decay in the $m d x$ mouse. A $60-80 \%$ reduction of the dystrophin transcript has been reported for the $m d x$ mouse compared with wild-type animals. ${ }^{22}$

Furthermore, absolute quantification allowed us to compare the molecular approaches of several reports that assessed exon skipping in $m d x$ mice. As Table 2 shows, the number of dystrophin RNA molecules used as template greatly differs from report to report ranging from 1100 to 17700 transcripts. The excessive number of amplification cycles (last two rows of Table 2) leads to a significant deviation from our digital array golden standard results $(P$-value $<0.02)$ (Figure 2$)$. We believe standardization to determine the exon skipping levels is needed to better understand the outcome of different treatments inducing exon skipping.

It has been suggested that exon skipping levels assessed by RT-PCR give an overestimation, because the smaller skipped products amplify more efficiently than the bigger non-skipped fragments. However, we show here that for 30 amplification cycles using a single round amplification, there is no preferential amplification of the shorter product. When comparing skipping values obtained with absolute and relative quantification via densitometry and bioanalyzer analysis for a single round PCR, we found that skipping values were very similar. Thus, apparently stochastic effects do not interfere with the measurements. Table 1 shows that most values that fall in a $25 \%$ error margin relative to the absolute quantification results from the digital array were obtained using either bioanalyzer or densitometer. Nonoverestimation is also underlined by the finding that a one round amplification (20/25/30 cycles) of isolated skipped and unskipped molecules in known skipping percentages led to a slight underestimation of real skipping percentages rather than an overestimation (Figure 4). However, when two rounds of amplification were used, skipping levels were generally overestimated. Nevertheless, since dystrophin is a lowly expressed gene, one round of amplification might not be sufficient when analyzing exon skipping in cultured cells or in muscles from severely affected patients. This will require further investigation.

We discarded MCA analysis because it was not sufficiently sensitive; this could be due to a contamination while purifying the skipped and unskipped templates from the agarose gel. However, 80 PCR cycles were performed on the isolated templates and only specific amplification product was observed (Supplementary Figure 1b), making this probably non-specific background.

Although digital array represents the golden standard we would like to underline that there are some limitations: some VIC to FAM channel bleeding was observed 
(Supplementary Figure 1a) with both the skipped or unskipped detecting probes. It is possible to correct for this by applying a threshold in this methodology. However, in real-time quantitative PCR analysis, when both skipped and unskipped transcripts are present in a single reaction, this bleeding will influence quantification. More importantly, the costs of the digital array analysis are much higher compared with a normal PCR, especially when analyzing many samples, although new digital arrays in which 48 samples can be analyzed in a single run are now available. Furthermore, the analysis of the curves cannot be standardized and is experiment specific. Nevertheless, results obtained from two different experiments can be compared because the quantification is absolute.

Exon skipping can be quantified using different techniques. However, quantification using a bioanalyzer or a densitometer after primary amplification seems the preferred method for $m d x$ muscle tissue derived samples, because the values obtained for low and high exon skipping levels are close to the actual values. These methods are more straightforward and significantly less expensive than digital array analysis, which serves as a golden standard. It is however possible that the optimal method(s) will differ for other exons and therefore methods to quantify the skipping of other (human) exons should be assessed individually, although as a more general rule exon-skipping levels should not be quantified using nested PCR.

Supplementary Information accompanies the paper on the Laboratory Investigation website (http://www.laboratoryinvestigation.org)

\section{ACKNOWLEDGEMENTS}

We thank Emmelien Aten (LUMC), Giovanni Perini (University of Bologna) and Marina Fabris (University of Ferrara) for helpful suggestions. The LUMC and the University of Ferrara are partners of the TREAT-NMD Network of excellence. This work was supported by Dutch Duchenne Parent Project to [A. A-R.]; ZonMw to [A. A-R.]; Telethon Italy [Grant number GGPU7011 to AF]; and the TREAT-NMD network of excellence.

\section{DISCLOSURE/CONFLICT OF INTEREST}

The authors declare no conflict of interest.

1. van Ommen GJ, van Deutekom J, Aartsma-Rus A. The therapeutic potential of antisense-mediated exon skipping. Curr Opin Mol Ther 2008;10:140-149. Review.

2. Aartsma-Rus A, Fokkema I, Verschuuren J, et al. Theoretic applicability of antisense-mediated exon skipping for Duchenne muscular dystrophy mutations. Hum Mutat 2009;30:293-299. Review.

3. van Deutekom JC, Janson AA, Ginjaar IB, et al. Local dystrophin restoration with antisense oligonucleotide PRO051. N Engl J Med 2007;357:2677-2686.
4. Kinali $M$, Arechavala-Gomeza V, Feng $L$, et al. Local restoration of dystrophin expression with the morpholino oligomer AVI-4658 in Duchenne muscular dystrophy: a single-blind, placebo-controlled, dose-escalation, proof-of-concept study. Lancet Neurol 2009;8: 918-928.

5. Heemskerk $\mathrm{HA}$, de Winter $\mathrm{CL}$, de Kimpe $\mathrm{SJ}$, et al. In vivo comparison of 2'-O-methyl phosphorothioate and morpholino antisense oligonucleotides for Duchenne muscular dystrophy exon skipping. J Gene Med 2009;11:257-266.

6. Arechavala-Gomeza V, Graham IR, Popplewell L, et al. Comparative analysis of antisense oligonucleotide sequences for targeted skipping of exon 51 during dystrophin pre-mRNA splicing in human muscle. Hum Gene Ther 2007;18:798-810.

7. Yin $\mathrm{H}$, Moulton HM, Seow $\mathrm{Y}$, et al. Cell-penetrating peptide-conjugated antisense oligonucleotides restore systemic muscle and cardiac dystrophin expression and function. Hum Mol Genet 2008;17: 3909-3918.

8. Ivanova GD, Arzumanov A, Abes $R$, et al. Improved cell-penetrating peptide-PNA conjugates for splicing redirection in HeLa cells and exon skipping in $m d x$ mouse muscle. Nucleic Acids Res 2008;36: 6418-6428.

9. Lu QL, Rabinowitz A, Chen YC, et al. Systemic delivery of antisense oligoribonucleotide restores dystrophin expression in body-wide skeletal muscles. Proc Natl Acad Sci USA 2005;102:198-203.

10. Doran $\mathrm{P}$, Wilton SD, Fletcher $\mathrm{S}$, et al. Proteomic profiling of antisenseinduced exon skipping reveals reversal of pathobiochemical abnormalities in dystrophic $m d x$ diaphragm. Proteomics 2009;9: 671-685.

11. Denti MA, Incitti T, Sthandier O, et al. Long-term benefit of adenoassociated virus/antisense-mediated exon skipping in dystrophic mice. Hum Gene Ther 2008;19:601-608.

12. Wu B, Lu P, Benrashid E, et al. Dose-dependent restoration of dystrophin expression in cardiac muscle of dystrophic mice by systemically delivered morpholino. Gene Therapy 2009;17: $132-140$.

13. Reed GH, Kent JO, Wittwer CT. High-resolution DNA melting analysis for simple and efficient molecular diagnostics. Pharmacogenomics 2007;8:597-608

14. Deligezer U, Esin Akisik E, Dalay $\mathrm{N}$, et al. A novel application of melting curves: utility of peak area calculation for relative methylation quantification. Clin Chem Lab Med 2007;45:867-873.

15. Zimmermann BG, Grill S, Holzgreve W, et al. Digital PCR: a powerful new tool for noninvasive prenatal diagnosis? Prenat Diagn 2008:28:1087-1093. Review.

16. Vitiello L, Bassi N, Campagnolo $\mathrm{P}$, et al. In vivo delivery of naked antisense oligos in aged $m d x$ mice: analysis of dystrophin restoration in skeletal and cardiac muscle. Neuromuscul Disord 2008;18:597-605.

17. Muir LA, Chamberlain JS. Emerging strategies for cell and gene therapy of the muscular dystrophies. Expert Rev Mol Med 2009;11:e18. Review.

18. Wilton SD, Lloyd F, Carville K, et al. Specific removal of the nonsense mutation from the $m d x$ dystrophin mRNA using antisense oligonucleotides. Neuromuscul Disord 1999;9:330-338.

19. Bremmer-Bout $M$, Aartsma-Rus $A$, de Meijer EJ, et al. Targeted exon skipping in transgenic hDMD mice: a model for direct preclinical screening of human-specific antisense oligonucleotides. Mol Ther 2004;10:232-240.

20. Mortazavi A, Williams BA, McCue $K$, et al. Mapping and quantifying mammalian transcriptomes by RNA-Seq. Nat Methods 2008;5:621-628.

21. Sommer SS, Cohen JE. The size distributions of proteins, mRNA, and nuclear RNA. Mol Evol 1980;15:37-57.

22. Rimessi $P$, Sabatelli $P$, Fabris $M$, et al. Cationic PMMA nanoparticles bind and deliver antisense oligoribonucleotides allowing restoration of dystrophin expression in the $m d x$ mouse. Mol Ther 2009;17:820-827. 